\title{
Odontogenic tumors: A collaborative study of 218 cases diagnosed over 12 years and comprehensive review of the literature
}

\author{
Ahmet-Ercan Sekerci ${ }^{1}$, Sinan Nazlım ${ }^{2}$, Meryem Etoz ${ }^{3}$, Kemal Denız ${ }^{2}$, Yasin Yasa ${ }^{4}$ \\ ${ }^{1}$ Assistant Professor, Department of Oral and Maxillofacial Radiology, Faculty of Dentistry, Erciyes University, Kayseri, Turkey \\ ${ }^{2}$ Department of Pathology, School of Medicine, Erciyes University, Kayseri, Turkey \\ ${ }^{3}$ Research Assistant, Department of Maxillofacial Radiology, Faculty of Dentistry, Erciyes University, Kayseri, Turkey \\ ${ }^{4}$ Research Assistant, Department of Maxillofacial Radiology, Faculty of Dentistry, Ataturk University, Erzurum, Turkey
}

Correspondence:

Erciyes University

Faculty of Dentistry

Department of Oral and Maxillofacial Radiology

Kayseri-Turkey

aercansekerci@hotmail.com

Received: 04/03/2014

Accepted: 10/04/2014
Sekerci AE, Nazlım S, Etoz M, Denız K, Yasa Y. Odontogenic tumors: A collaborative study of 218 cases diagnosed over 12 years and comprehensive review of the literature. Med Oral Patol Oral Cir Bucal. 2015 Jan 1;20 (1):e34-44.

http://www.medicinaoral.com/medoralfree01/v20i1/medoralv20ilp34.pdf

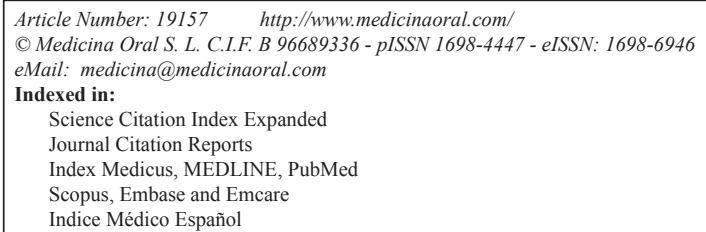

\begin{abstract}
Objectives: The objective of this study was to analyze the frequency and distribution of odontogenic tumors (OTs) in the Cappadocia region of Turkey, and to compare the findings with those reported in the literature.

Study Design: The records of the Oral and Maxillofacial Surgery and Pathology Departments at Erciyes University, with histologic diagnosis of odontogenic tumors (based on the World Health Organization classification, 2005), over a 12-year period, were analyzed. The relative frequency of different types of tumors was also analyzed and compared with the literature.

Results: OTs in the present study constituted $2.74 \%$ of all the 7,942 registered biopsies. A total of 218 cases of OTs were collected and reviewed. Of these, $(94.04 \%)$ were benign and $(5.96 \%)$ were malignant. The mandible was the most commonly affected anatomic location, with 170 cases $(77.9 \%)$. Ameloblastoma with a predilection for the posterior mandible was the most frequent odontogenic tumor (30.28\%), followed by keratocystic odontogenic tumor (19.5\%), odontoma (13.4\%), and odontogenic myxoma (8.5\%).

Conclusions: OTs are rare neoplasms and appear to show geographic variations in the world. In Cappadocia, Turkey, they are more common in the mandible, with ameloblastoma followed by keratocystic odontogenic tumors with the incidences observed in the present study being similar to those of previous studies from Asia and Africa, and in contrast to those reported from American countries.
\end{abstract}

Key words: Odontogenic tumors, WHO classification, prevalence, jaws. 


\section{Introduction}

Odontogenic tumors (OTs) constitute a heterogeneous group of lesions, arising from the tooth-producing tissues or its remnants (1). From a biological point of view, some of these lesions represent hamartomas with varying degrees of differentiation, while the rest are benign or malignant neoplasms with variable aggressiveness and potential to develop metastasis (2). OTs are rare lesions of the mandible and maxilla that must be considered as a part of the differential diagnosis of lesions that occur in the jaws (3). In humans, tumors of the odontogenic tissues are comparatively rare, comprising about $1 \%$ of all jaw tumors (4).

The first internationally accepted classification system for OTs was published in 1971 by the World Health Organization (WHO), which was reviewed and updated in 1992 and in 2005 (2).

Knowledge of their epidemiology and clinical presentation is essential, and retrospective studies have been carried out in Asia (5-16), Africa (17-30), Europe (31-33), North America (3,34-40), and South America (41-49) to describe these lesions. The geographic distribution of these lesions is variable, mainly because of high genetic and cultural diversity (41). Their etiology is unknown and the majority develops without an apparent cause (30).

It is very important to form a set of criteria such as sex, age, and location of lesion, in the management of OTs. Epidemiological studies are crucial because they allow us to establish more precisely the occurrence of OTs in different populations, which in turn helps in the making of a provisional diagnosis and further planning of the biopsy based on the clinical and radiographic features. It also aids in patient counseling and scheduling of treatment (14).

Furthermore, there is no information available in the English-language literature on the relative frequency of OTs in Turkey or, particularly, in the Cappadocia region, according to the $2005 \mathrm{WHO}$ classification. The purpose of the present study was to determine the relative prevalence of different types of OTs and to determine the relative incidence of different OTs in the world population through analysis of published studies and statistics, and by comparing these data with each other and with those already reported in the literature.

\section{Material and Methods}

In the present study, the surgical histopathology records of the Departments of Oral Pathology, Faculty of Medicine and Oral and Maxillofacial Surgery, Faculty of Dentistry, Erciyes University were reviewed retrospectively from August 2001 to January 2013. They were tabulated and systematically analyzed to assess the frequency of occurrence based on age, sex, anatomical site and type.

Hematoxylin and eosin stained sections were reviewed to confirm or to correct a previous histological diagnosis according to the criteria suggested for the 2005 WHO classification. The independ $\neg$ ent opinions of two examiners were compared to reach the final diagnosis and, in cases of doubt, we consulted another expert oral pathologist to obtain a diagnosis by consensus.

A total of 218 cases of OTs were collected and reviewed. The literature was retrieved using Pubmed in English only. Recurrent tumors were considered as a single case. With regard to site distribution, the maxilla was divided into three anatomic regions: anterior, premolar and molar; and the mandible was divided into three anatomic regions: anterior, premolar, molar/ ramus. Data were analyzed using SPSS software (version 11.5; SPSS, Inc, Chicago, IL).Tests were considered statistically significant when the p-value was $<0.05$.

\section{Results}

From total of 7,942 oral and maxillofacial biopsies registered during the 15-year period from 1998 to 2013, 218 cases of odontogenic tumors were found. The most frequent lesion was ameloblastoma (AME) (30.28\%), followed by keratocystic odontogenic tumor (KCOT) (26.15\%) (Table 1). The proportion of benign to malignant lesions was 15.8:1. Taken together, AME, KCOT, odontoma (OD), and calcifying epithelial odontogenic tumour (CEOT) corresponded to nearly $78 \%$ of the cases. There were no statistically significant differences between the ameloblastoma and keratocystic odontogenic tumor groups. In male patients, KCOT followed by AME and calcifying epithelial odontogenic tumor (CEOT) were the most common lesions; in female patients, AME, followed by KCOT and OD, were the most frequent OTs (data shown in table 1). (Examples of odontogenic tumors diagnosed with histopathological examination were shown in figure 1. Examples of cropped panoramic radiographs of patients with odontogenic tumors were shown in figure 2).

The other tumors comprised less than $6 \%$ of the series. An almost equal gender distribution was observed; with a slight predominance of males (the sample comprised $110(50.5 \%)$ males and 108 (49.5\%) females). Statistical analysis revealed no significant difference in the distribution of OT in relation to gender. AME was the only benign tumor found in patients over than 80 years of age. The age of the patients ranged from 10 years to 84 years, with a mean age of 34.52 years. The majority of cases were distributed between the age of 20 and 49 years with a peak incidence in the fourth decade of life (Table 2).

The anatomical sites of all cases are also presented in table 1. In general, the mandible was the most frequently affected site, corresponding to $77.9 \%$ of the cases, while the maxilla was affected in $22.1 \%$ of the cases. The most frequently affected area was the mandibular molar/ramus 


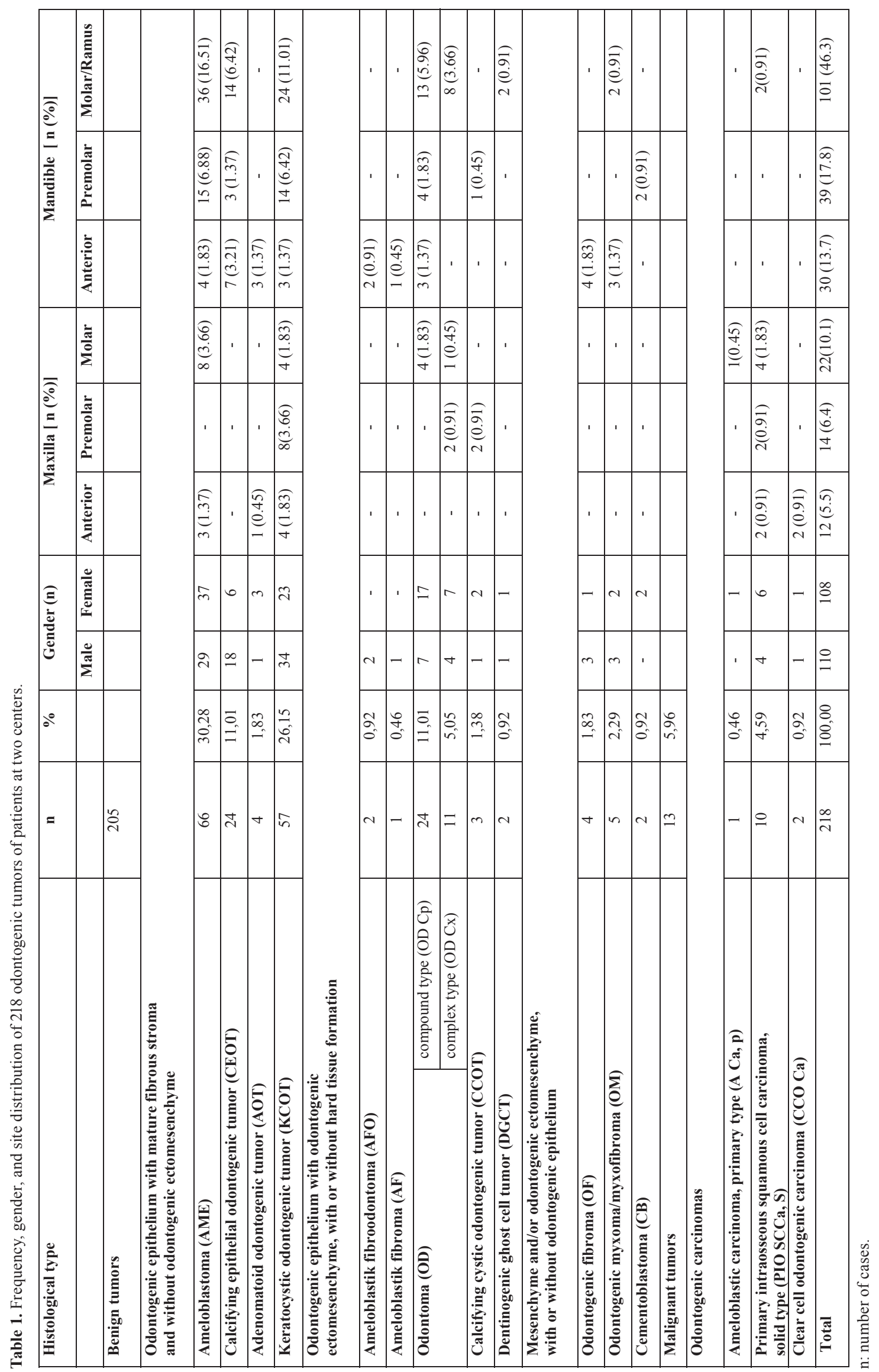



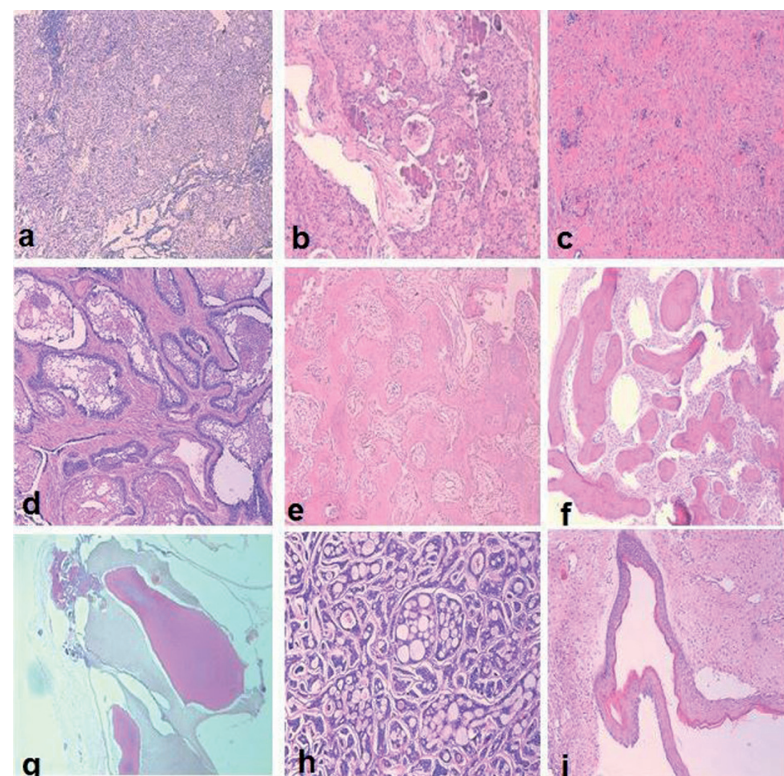

g

Fig. 1. Examples of odontogenic tumors diagnosed with histopathological examination. a) Adenomatoid odontogenic tumor, H\&Ex100; b) Calcifying epithelial odontogenic tumor, H\&Ex100; c) Odontogenic fibroma, H\&Ex100; d) Ameloblastoma, H\&Ex100; e) Cementoblastoma, H\&Ex100; f) Komplex odontoma, H\&Ex100; g) Kompound odontoma, H\&Ex40; h) Ameloblastic carcinoma, H\&Ex100; i) Keratocystic odontogenic tumor, H\&Ex100.

\section{Discussion}

The fact that most OTs remain painless throughout the course of the disease is the main reason that patients do not present until the tumors have reached enormous sizes (17). Knowing the frequency and basic clinical features of OTs is important because this allow us to establish more precisely the expression of these lesions in diverse populations, which in turn helps to identify the groups at risk and possible factors associated with their development, as well as to develop more precise differential diagnoses (2).

In the present study, the relative frequency of odontogenic tumors was $2.74 \%$ of the total biopsied specimens recorded between August 1998 and January 2013. This incidence is similar to what has been reported in other studies, as they represent less than $3 \%$ of oral and maxillofacial specimens studied in North American $(35,39)(1.55 \%)$, South American $(41,42,44,45)(1.82 \%)$, and European series $(32,33)(0.74 \%)$. On the other hand, in Asia and Africa OT comprise from 3.9\% to $9.6 \%$ of all oral lesions $(8,9,20)$, although an Iranian series (7) had a frequency of $1.9 \%$.

This study confirms that benign tumors (94.4\%) are the most frequently seen OT; however, malignant OT rep-
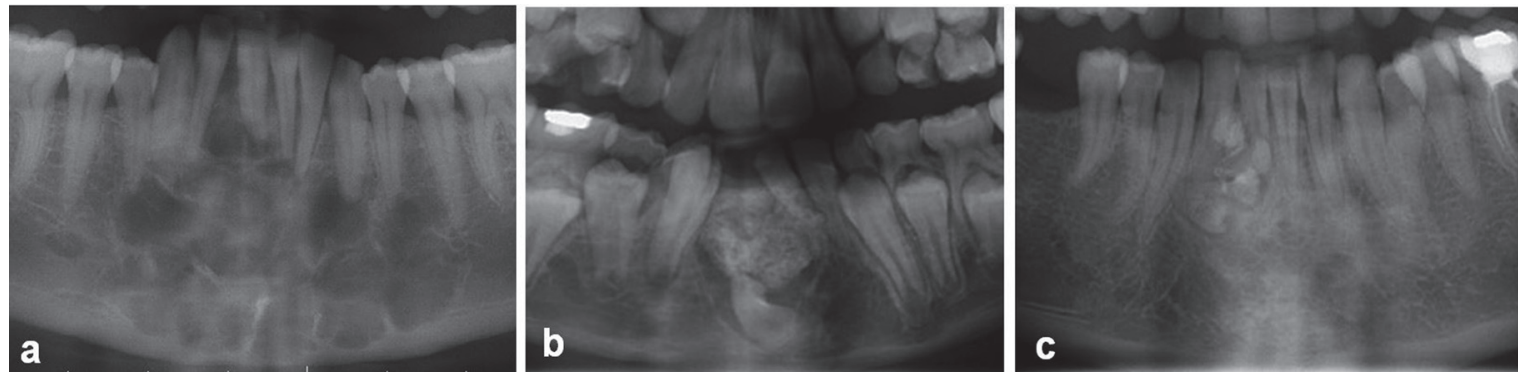

Fig. 2. Examples of cropped panoramic radiographs of patients with odontogenic tumors: a) Odontogenic myxoma, b) Ameloblastik fibro-odontom; c) Odontoma.

segment, mainly by AME. The youngest patient who presented with the lesion was 10 years old and the oldest was 84 years old. The single case of ameloblastic fibroma affected a 14-year-old male patient. The lesion was located in the anterior mandible. There were 2 cases of dentinogenic ghost cell tumor (DGCT), which is a new entity according to the 2005 WHO classification.

Malignant OT, ameloblastic carcinoma primary type (A $\mathrm{Ca}, \mathrm{p}$ ), primary intraosseous squamous cell carcinoma (PIO SCCa, S), and clear cell odontogenic carcinoma $(\mathrm{CCO} \mathrm{Ca})$ were found more frequently in the maxilla. In the upper jaw, PIO SCCa, S was the most common lesion, mainly observed in the molar region, followed by $\mathrm{CCO} \mathrm{Ca}$, mainly in the anterior region. Most malignant OTs also predominantly occurred in patients older than 40 years (Table 2 ). resented 5.6\% in the present series. This frequency of malignant tumors is only similar to those reported in China $(8,12)$, but it is higher than that those published in most other series $(23,39,40,42,44-48)$.

In studies using the new 2005 WHO classification, the most frequent OTs follow the sequence: ameloblastomas (30.28\%), KCOT (26.15\%), and odontomas (16.06\%) (Table 3). Studies that employed the 1992 classification usually reported ameloblastomas as the prevalent OT, followed by odontomas and odontogenic myxomas (Table 4). This regional difference has been attributed to the asymptomatic nature of many odontomas and consequent lack of professional management rather than genetic or environmental differences among these populations $(19,20,35,44)$. The present study found ameloblastoma to be the most frequent odontogenic tumor, 
Table 2. Distribution of odontogenic tumors in different age groups.

\begin{tabular}{|c|c|c|c|c|c|c|c|c|c|c|c|}
\hline & & & & Age gro & Ip (year & & & & & & \\
\hline & Sex ratio & $10-19$ & 20-29 & 30-39 & $40-49$ & $50-59$ & $60-69$ & $70-79$ & 80-89 & Total & $\%$ \\
\hline Benign & & & & & & & & & & 205 & 94.04 \\
\hline AME & $1: 1.3$ & 6 & 12 & 7 & 13 & 7 & 4 & 11 & 6 & 66 & 30.28 \\
\hline CEOT & 1: 0.3 & - & - & 2 & 12 & 11 & 6 & - & - & 24 & 11.01 \\
\hline AOT & $1: 3$ & - & 2 & 2 & & & & - & - & 4 & 1.83 \\
\hline KCOT & 1:0.7 & - & 15 & 12 & 14 & 10 & 7 & - & - & 57 & 26.15 \\
\hline AFO & - & 1 & 1 & - & - & - & - & - & - & 2 & 0.92 \\
\hline $\mathbf{A F}$ & - & 1 & - & - & - & - & - & - & - & 1 & 0.46 \\
\hline OD Cp & $1: 2.4$ & 11 & 4 & 6 & 2 & 1 & - & - & - & 24 & 11.01 \\
\hline OD Cx & $1: 1.8$ & 1 & 3 & 4 & 1 & 2 & - & - & - & 11 & 5.05 \\
\hline CCOT & $1: 2$ & - & - & 1 & 2 & - & - & - & - & 3 & 1.38 \\
\hline DGCT & $1: 1$ & - & - & 1 & 1 & - & - & - & - & 2 & 0.92 \\
\hline OF & $1: 0.3$ & - & - & - & 3 & 1 & - & - & - & 4 & 1.83 \\
\hline OM & $1: 0.7$ & 2 & - & 1 & 1 & 1 & - & - & - & 5 & 2.29 \\
\hline CB & - & - & - & 2 & - & - & - & - & - & 2 & 0.92 \\
\hline Malignant & & & & & & & & & & 13 & 5.96 \\
\hline A Ca.p & - & - & - & - & - & - & 1 & - & - & 1 & 0.46 \\
\hline PIO SCCa & $1: 1.5$ & - & - & - & 5 & 1 & 3 & - & 1 & 10 & 4.59 \\
\hline $\mathrm{CCO} \mathrm{Ca}$ & $1: 1$ & - & - & - & - & & 1 & - & 1 & 2 & 0.92 \\
\hline & & & & & & & & & & 218 & \\
\hline
\end{tabular}

n: number of cases

accounting for $30.28 \%$, followed by KCOT (26.15\%), odontoma (16.06\%), and CEOT (11.01\%). There were no statistically significant differences among the ameloblastoma and KCOT and odontoma groups. These results are comparable with the corresponding data reported by Jing et al. (10), Tawfik et al. (17) and Osterne et al. (46). Ameloblastoma is reported to be the most frequent lesion in Chinese, Egyptian and Brazilian populations, followed by KCOT and odontoma.

The high frequency of AME and low frequency of odontoma are consistent with data from Tanzania (21), Nigeria $(20,25,44)$, and Sri Lanka (11) whereas studies from the USA (35), Canada (22), Chennai-India (14) and Estonia (9), stated that odontoma occurs more frequently. These discrepancies in the number of odontomas being less in their populations in comparison with others are probably the result of geographic variation, but it should be mentioned that the incidence of odontoma in some countries was probably underestimated due to the unique clinical features of this tumor and insufficient hospital management (32). Most of these tumors exhibit self-limited growth and do not cause clinical symptoms. Many patients do not think it is necessary to consult a general dentist or even an oral and maxillofacial surgeon. Treatment in many cases was performed in the office and the cases were not recorded or sent for histopathological confirmation $(9,17)$.

The present study showed that AME was the most frequent OT, occurring mainly in the posterior region of the mandible. This is similar to other studies reported from Japan (14), Iran (7), India (14,15), Srilanka (16), Africa $(17,18,20,23,25,30)$, Turkey (33), Hong Kong (13), and China $(10,12)$, but in contrast to those reported from Canada (40), Chile (44), USA (35), Chennai (14) and Mexico $(34,39)$, where odontoma is reported as the most common odontogenic tumor. This also strengthens the belief that ameloblastomas are more common in Asians and Africans compared with Caucasians. A study form Brazil reported that ameloblastoma diagnosis exhibits no gender predilection (43). Reichart et al. (49) in an extensive review of all of the cases reported in the literature, reported the average age of initial diagnosis in industrialized countries to be 39.1 years compared with 27.7 years from developing countries. Sriram et al (9) reported that almost $95 \%$ of ameloblastomas were located in the mandible, with a very high mandible to maxilla ratio (18.1:1). This is very high compared with the ratios reported by earlier studies (Table 5). Reichart et al. (49) in their extensive review of 3,677 cases of ameloblastoma, found the ratio to be around 5.4:1. In 


\begin{tabular}{|c|c|c|c|c|c|c|c|c|c|c|c|c|c|c|c|c|c|c|c|c|c|c|c|c|c|}
\hline 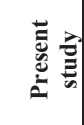 & 공 & & $\begin{array}{l}\text { तิ } \\
0 \\
0 \\
0 \\
0\end{array}$ & | & $\begin{array}{l}a \\
\\
y \\
\vdots\end{array}$ & 它 & (ְ) & 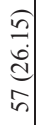 & ลิ & $\begin{array}{l}\frac{6}{+} \\
\stackrel{+}{8} \\
-\end{array}$ & 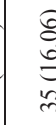 & 1 & $\stackrel{\infty}{m}_{m}^{\infty}$ & 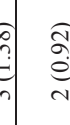 & 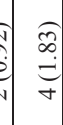 & 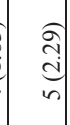 & 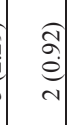 & $\mid \begin{array}{c}0 \\
\mathfrak{o} \\
0 \\
-\end{array}$ & I & $\begin{array}{l}\underset{\hat{n}}{\mathfrak{d}} \\
\stackrel{0}{\sigma}\end{array}$ & ลิ & 1 & 1 & I & 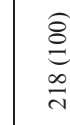 \\
\hline 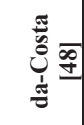 & & $\stackrel{\varrho}{\sharp}$ & $\begin{array}{l}\infty \\
\grave{d} \\
0 \\
0\end{array}$ & | & 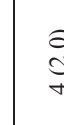 & & : & 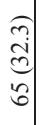 & $\underset{\sim}{\stackrel{\theta}{\Xi}}$ & $\underset{\sigma}{\stackrel{d}{d}}$ & $\begin{array}{l}7 \\
\infty \\
\infty\end{array}$ & 1 & 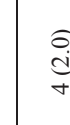 & $\begin{array}{l}\tilde{j} \\
\dot{s}\end{array}$ & 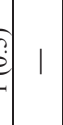 & $\begin{array}{l}\tilde{n} \\
\dot{\jmath} \\
\sigma\end{array}$ & 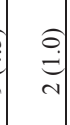 & $\mid \begin{array}{c}n \\
0 \\
n \\
n\end{array}$ & I & $\stackrel{\hat{n}}{\hat{\theta}}$ & $\stackrel{n}{\varrho}$ & 1 & 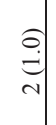 & 1 & $\begin{array}{l}\vec{\sim} \\
\stackrel{-}{1}\end{array}$ \\
\hline 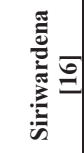 & & @ & $\begin{array}{l}0 \\
0 \\
0 \\
0 \\
0 \\
\infty \\
0\end{array}$ & s. & $\begin{array}{l}n \\
7 \\
27\end{array}$ & 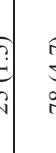 & $\begin{array}{l}\text { E. } \\
+ \\
\infty\end{array}$ & 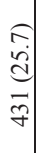 & $\underset{a}{\hat{e}}$ & ڤn & $\begin{array}{l}7 \\
0 \\
8\end{array}$ & 1 & $\underset{\sim}{\stackrel{f}{\Xi}}$ & f. & $\stackrel{f}{\stackrel{f}{\circ}}$ & 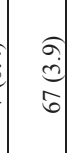 & $\begin{array}{l}0 \\
0 \\
0 \\
-1\end{array}$ & $\mid \begin{array}{c}\hat{a} \\
\stackrel{n}{m}\end{array}$ & : & $\begin{array}{l}0 \\
\stackrel{6}{0} \\
0 \\
0\end{array}$ & ڤึ) & I & $\mid \begin{array}{c}0 \\
\vdots \\
0 \\
-1\end{array}$ & $\stackrel{\widehat{E}}{\stackrel{\theta}{0}}$ & 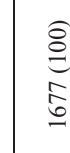 \\
\hline 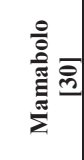 & & @ & $\begin{array}{l}6 \\
\dot{8} \\
8 \\
0\end{array}$ & 1 & $\frac{\sqrt{3}}{\vec{m}}$ & 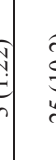 & הֶ) & 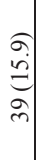 & $\stackrel{\sqrt[n]{+}}{=}$ & I & $\begin{array}{l}\sigma \\
\infty \\
\infty \\
\infty\end{array}$ & & & & 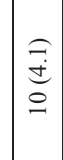 & 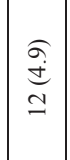 & 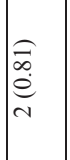 & $\underset{\sigma}{\sigma}$ & I & | & 1 & 1 & 1 & | & $\underset{⿱ 乛 ⿻}{+}$ \\
\hline 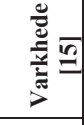 & & @্ & $\begin{array}{l}\widehat{\alpha} \\
\dot{q}+\dot{q} \\
\sigma \\
\end{array}$ & 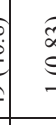 & $\begin{array}{l}\widehat{\infty} \\
\infty \\
0 \\
-\end{array}$ & 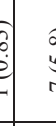 & 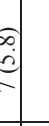 & $\begin{array}{l}n \\
0 \\
0 \\
y\end{array}$ & $\stackrel{\widetilde{\infty}}{\stackrel{\infty}{\infty}}$ & I & $\begin{array}{l}0 \\
\\
\end{array}$ & 1 & $\begin{array}{l}\widehat{\infty} \\
\stackrel{\infty}{\Theta} \\
-\end{array}$ & 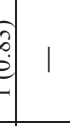 & 1 & 1 & $\begin{array}{c}\widehat{\widehat{O}} \\
\stackrel{0}{0} \\
0\end{array}$ & 1 & 1 & | & I & 1 & 1 & | & $=$ \\
\hline $\begin{array}{l}\bar{\Xi} \\
\stackrel{\vec{\Xi}}{0}\end{array}$ & $\vec{i}$ & $\stackrel{\varrho}{Ð}$ & 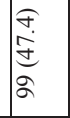 & 1 & 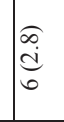 & 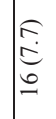 & 守 & & $\underset{\sim}{\stackrel{\sigma}{\rightleftarrows}}$ & I & 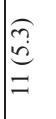 & I & 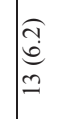 & | & & $\begin{array}{c}\hat{n} \\
\stackrel{n}{r} \\
r\end{array}$ & बิ & 1 & I & I & | & 1 & 1 & | & 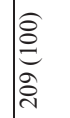 \\
\hline 产导 & 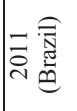 & @ & 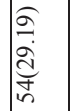 & I & 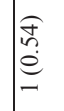 & 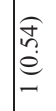 & $\approx$ & & $\frac{\widehat{\sigma}}{\mathrm{d}}$ & I & 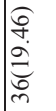 & | & $\mid \begin{array}{l}\widehat{\alpha} \\
\stackrel{\tilde{n}}{\curvearrowright}\end{array}$ & 1 & $\begin{array}{l}\infty \\
\infty \\
\infty \\
\stackrel{2}{r}\end{array}$ & 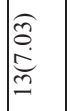 & $\begin{array}{l}\widehat{\infty} \\
\stackrel{\infty}{2} \\
\stackrel{2}{2}\end{array}$ & 1 & | & 1 & 1 & | & 1 & & 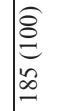 \\
\hline 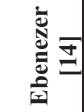 & 율 & @ & 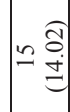 & । & 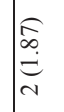 & $\begin{array}{l}\widehat{\infty} \\
\stackrel{d}{d} \\
m\end{array}$ & $m$ & $=$ & 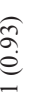 & 仝 & 㝴 & 1 & 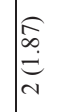 & | & $\widehat{\widehat{e}}$ & 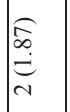 & $\widehat{\widehat{Q}}$ & 1 & | & I & | & | & 1 & 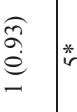 & 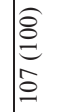 \\
\hline 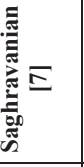 & 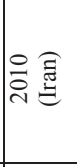 & @ & 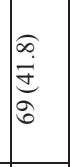 & I & 1 & $\begin{array}{l}\widehat{\bar{a}} \\
\stackrel{2}{a}\end{array}$ & | & & $\frac{\substack{f \\
d}}{d}$ & 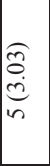 & 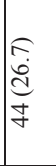 & 它 & 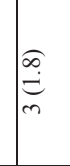 & I & 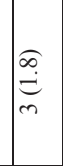 & 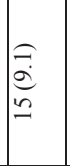 & $\begin{array}{l}\widehat{c} \\
\dot{c} \\
\dot{c} \\
\text { in }\end{array}$ & 1 & | & I & | & | & 1 & $\mid$ & 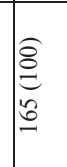 \\
\hline 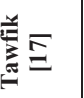 & 䝃 & @ & 等 & 1 & $\begin{array}{l}\hat{e} \\
\stackrel{m}{n}\end{array}$ & $\underset{\tilde{e}}{\stackrel{c}{n}}$ & 现 & & $\underset{\text { d্ }}{\stackrel{f}{d}}$ & 1 & 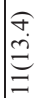 & | & 1 & 1 & 1 & $\mid \begin{array}{l}\hat{n} \\
\stackrel{\infty}{r} \\
\stackrel{2}{n}\end{array}$ & $\begin{array}{l}\hat{E} \\
\stackrel{\tilde{m}}{n}\end{array}$ & I & $\stackrel{\overbrace =}{\Xi}$ & $\stackrel{\text { d }}{\text { d }}$ & 1 & | & 1 & | & 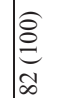 \\
\hline - & ì & @ & 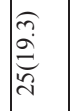 & I & 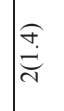 & $\underset{\text { J }}{\stackrel{\Im}{ \pm}}$ & $\begin{array}{c}\sigma \\
\infty \\
\infty \\
\infty \\
\infty \\
i n\end{array}$ & & | & 1 & $\begin{array}{l}\widehat{\infty} \\
\dot{0} \\
\tilde{c} \\
y \\
y\end{array}$ & 1 & 1 & 1 & 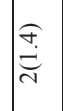 & $\begin{array}{l}\infty \\
\dot{\omega} \\
\infty \\
\infty\end{array}$ & | & 1 & | & I & | & | & 1 & & 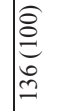 \\
\hline 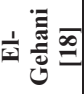 & 商 & 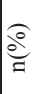 & 욜 & 1 & $\underset{\sim}{\stackrel{\Upsilon}{*}}$ & 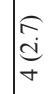 & $m$ & & $\underset{\sim}{\stackrel{\widehat{C}}{\rightleftarrows}}$ & | & กิร & I & 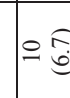 & $\begin{array}{c}c \\
\dot{0}\end{array}$ & I & 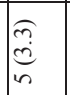 & I & 1 & | & 1 & | & | & 1 & $=\underset{\stackrel{f}{S}}{\subseteq}$ & 邑 。 \\
\hline$\frac{\infty}{\varrho}$ & 产 & 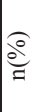 & 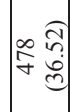 & 1 & $\begin{array}{l}0 \\
0 \\
0 \\
0 \\
0\end{array}$ & $\begin{array}{l}\mathscr{Q} \\
\stackrel{d}{d} \\
\grave{\lambda}\end{array}$ & in & & $\begin{array}{l}\widehat{\alpha} \\
\hat{e} \\
\underline{\underline{n}}\end{array}$ & 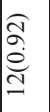 & 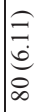 & | & 命 & $\begin{array}{l}\infty \\
\infty \\
e \\
e \\
n \\
n\end{array}$ & 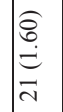 & 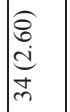 & 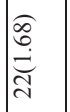 & 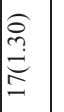 & | & $\begin{array}{l}\underset{f}{d} \\
\stackrel{d}{g} \\
\text { de }\end{array}$ & $\begin{array}{l}\widehat{\vec{b}} \\
\stackrel{\infty}{\infty}\end{array}$ & స̂ન & & | & बेळ \\
\hline 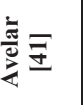 & 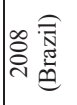 & $\stackrel{\varrho}{=}$ & $\begin{array}{l}\stackrel{a}{a} \\
\underset{d}{d} \\
\text { in }\end{array}$ & $\underset{f}{\stackrel{f}{e}}$ & 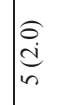 & $\begin{array}{l}\mathcal{f} \\
\stackrel{n}{n} \\
\end{array}$ & 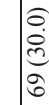 & & $\stackrel{F}{E}$ & 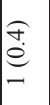 & 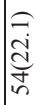 & | & 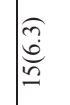 & | & | & $\begin{array}{l}\widehat{3} \\
0 \\
\underline{0} \\
\underline{n}\end{array}$ & $\stackrel{\overbrace{}}{\stackrel{f}{z}}$ & 1 & | & 1 & | & 1 & I & | & 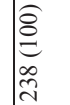 \\
\hline 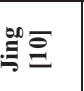 & 产 & $\grave{g}^{\circ}$ & छ) $\stackrel{\hat{q}}{\stackrel{\rho}{q}}$ & $\int_{\substack{n \\
e_{n}}}$ & $\cong 0$ & $\widehat{C}$ & $\begin{array}{l}\infty \\
\infty \\
\infty\end{array}$ & 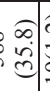 & త্ড & $\mid \begin{array}{c}\hat{y} \\
\hat{y} \\
\hat{y}\end{array}$ & 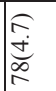 & 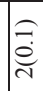 & 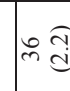 & 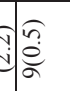 & $\widehat{\widehat{n}} \overline{\hat{e}}$ & $\therefore \stackrel{0}{+}$ & $m \vec{d}$ & 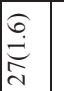 & I & $\pm \hat{\mathscr{e}}$ & 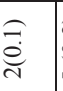 & 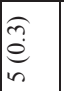 & & 1 & ț \\
\hline & & & 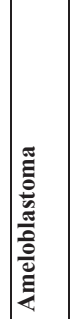 & 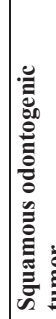 & 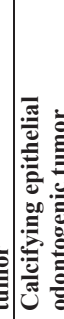 & 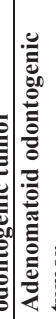 & 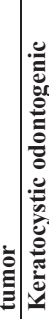 & & 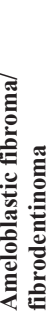 & & 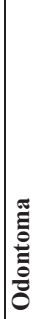 & 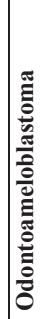 & 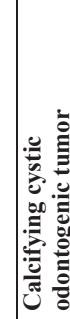 & 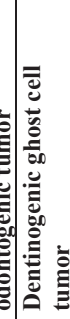 & 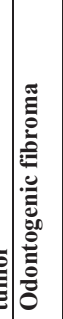 & 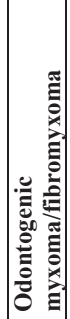 & 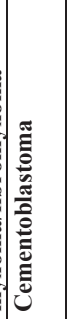 & 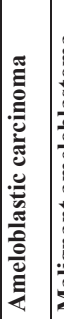 & 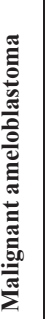 & 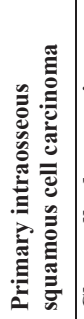 & 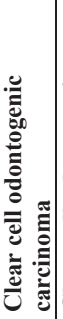 & 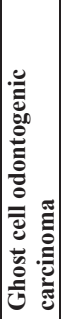 & 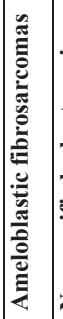 & 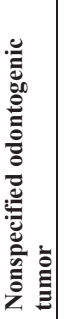 & \\
\hline
\end{tabular}




\begin{tabular}{|c|c|c|c|c|c|c|c|c|c|c|c|c|c|c|c|c|c|c|c|}
\hline$\stackrel{50}{*}$ & $0^{\circ}$ & $\overrightarrow{0}$ & $\tilde{0}$ & $\begin{array}{l}n \\
i n\end{array}$ & $\stackrel{\sim}{-}$ & $\stackrel{m}{i}$ & $\overrightarrow{6}$ & $\begin{array}{l}0 \\
:\end{array}$ & $\stackrel{m}{-}$ & & & $\stackrel{?}{\rightarrow}$ & $\begin{array}{r}t \\
\dot{r}\end{array}$ & $\stackrel{\nabla}{0}$ & $\exists$ & & & & $\hat{o}$ \\
\hline 已ึ & $\otimes^{\circ}$ & $\stackrel{\sim}{0}$ & $\sim$ & $\hat{\circ}$ & $a$ & 1 & 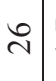 & $\stackrel{-}{-}$ & $\begin{array}{l}\infty \\
\stackrel{0}{0} \\
0^{-1}\end{array}$ & $\stackrel{+}{i}$ & I. & 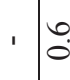 & 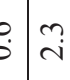 & $\grave{0}$ & $\stackrel{9}{-}$ & - & $\stackrel{9}{-}$ & d & $\begin{array}{l}\tilde{\alpha} \\
\infty \\
0\end{array}$ \\
\hline$\sum$ & $0^{\circ}$ & $\begin{array}{l}0 \\
i \\
i\end{array}$ & $\begin{array}{l}\dot{0} \\
\dot{n}\end{array}$ & $\simeq$ & $\begin{array}{c}m \\
\infty \\
\infty\end{array}$ & 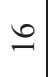 & $\stackrel{+}{\infty}$ & $\begin{array}{l}\infty \\
\infty \\
\infty\end{array}$ & $\simeq$ & $\stackrel{f}{+}$ & -10 & $\begin{array}{c}9 \\
= \\
\end{array}$ & $\vdots \bar{a}$ & $\approx$ & $\ddot{i}$ & $\stackrel{\dot{f}}{+}$ & 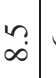 & 0 & $\begin{array}{l}\stackrel{0}{\circ} \\
\text { i. }\end{array}$ \\
\hline 考 & $0^{\circ}$ & $\stackrel{n}{-}$ & in & \begin{tabular}{|l|} 
\\
\end{tabular} & $\stackrel{-}{-}$ & $\begin{array}{l}0 \\
- \\
-\end{array}$ & $\vec{\circ}$ & $n$ & I & $r$ & $\underset{-}{-}: 9$ & 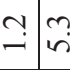 & 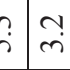 & $\stackrel{n}{-}$ & $a$ & $\dot{0}$ & $\stackrel{\check{r}}{\check{r}}$ & $\delta$ & $\stackrel{+}{\circ}$ \\
\hline ర్ & $0^{\circ}$ & $\left|\begin{array}{l}\infty \\
i\end{array}\right|$ & ' & $\stackrel{\vec{i}}{i}$ & 1 & $\stackrel{0}{-}$ & $\dot{o}$ & $\begin{array}{l}\bullet \\
0\end{array}$ & $\stackrel{?}{-}$ & & $\stackrel{-}{=}$ & $\because$ & $\begin{array}{c}c \\
i\end{array}$ & $\overrightarrow{0}$ & $\begin{array}{l}n \\
\sim \\
i\end{array}$ & $\stackrel{+}{+}$ & $\dot{m}$ & & กֶ. \\
\hline$\overleftrightarrow{0}$ & $0^{\circ}$ & $\stackrel{\leftrightarrow}{0}$ & ' & 1 & 1 & 1 & $?$ & ' & ' & ' & $\begin{array}{ll}1 & 1\end{array}$ & 19 & $? \stackrel{\infty}{\infty}$ & 1 & 1 & 1 & ' & ' & \\
\hline ठิ & $0^{\circ}$ & $\mid \begin{array}{c}\vec{b} \\
\infty \\
i\end{array}$ & in & $\stackrel{\sim}{\sim}$ & $\mid \begin{array}{l}\infty \\
\dot{n} \\
n \\
n\end{array}$ & & $\widehat{\sigma}$ & ヶ & 离 & in & \begin{tabular}{l|l}
$\stackrel{0}{i}$ &
\end{tabular} & 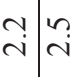 & $i \curvearrowleft$ & $\left|\begin{array}{c}0 \\
i \\
i\end{array}\right|$ & $\dot{\vec{i}}$ & $\stackrel{\leftrightarrow}{+}$ & $\begin{array}{l}\stackrel{a}{n} \\
\dot{n}\end{array}$ & 0 & $\stackrel{\curvearrowright}{i}$ \\
\hline $\begin{array}{l}0 \\
\text { 至 } \\
2\end{array}$ & $0^{\circ}$ & Na & $\stackrel{\nabla}{\dot{r}}$ & 1 & $\grave{0}$ & $\begin{array}{l}\infty \\
0 \\
0\end{array}$ & $?$ & $\stackrel{\sim}{i}$ & ' & 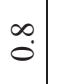 & & $\begin{array}{c}? \\
0\end{array}$ & 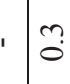 & 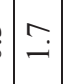 & 1 & $\stackrel{n}{-}$ & $\begin{array}{l}\bullet \\
+\end{array}$ & & ֻै \\
\hline$\frac{2}{\frac{2}{4}}$ & $0^{\circ}$ & $\stackrel{0}{0}$ & 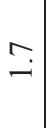 & $\begin{array}{l}\stackrel{n}{+} \\
+\end{array}$ & $\stackrel{n}{-}$ & $\vec{m}$ & $\mid$ & $\begin{array}{l}0 \\
:\end{array}$ & $\stackrel{0}{-}$ & $\stackrel{0}{-}$ & $\stackrel{-}{c}$ & $\vec{m} \mid N$ & $v \stackrel{\infty}{-}$ & $\stackrel{0}{-}$ & $\stackrel{n}{-}$ & ¿. & 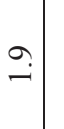 & & $\stackrel{\overbrace{}}{\overbrace{}}$ \\
\hline$\frac{1}{0}$ & $0^{\circ}$ & $\stackrel{\infty}{-}$ & $\hat{m}$ & ஸु & $\stackrel{⿱}{r}$ & $\leadsto$ & $\begin{array}{c}m \\
\infty \\
\infty\end{array}$ & $\begin{array}{l}0 \\
0 \\
0\end{array}$ & $\stackrel{\oplus}{-}$ & $\hat{\infty}$ & \begin{tabular}{l|l}
$\hat{\sigma}$ & $\alpha$ \\
$\varrho$ &
\end{tabular} & $\begin{array}{lll}\infty & \stackrel{n}{i} \\
\end{array}$ & $? \stackrel{\infty}{\infty}$ & 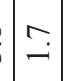 & $\vec{a}$ & $\stackrel{n}{2}$ & $\underset{n}{n}:$ & $\simeq$ & $a$ \\
\hline 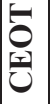 & $0^{\circ}$ & $\grave{o}$ & $\stackrel{ナ}{-}$ & $\ddot{0}$ & $\hat{o}$ & $\stackrel{0}{-}$ & $\hat{o}$ & $\begin{array}{l}0 \\
:\end{array}$ & $\stackrel{?}{-}$ & r & $\stackrel{-}{-}-$ & $-\stackrel{0}{-}$ & 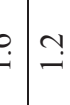 & $\begin{array}{l}n \\
0\end{array}$ & $\dot{o}$ & $?$ & $\stackrel{?}{-}$ & & $\begin{array}{l}\tilde{D} \\
0 \\
0\end{array}$ \\
\hline D্ & $0^{\circ}$ & ' & ' & -1 & 1 & 1 & $\stackrel{\nabla}{0}$ & $\begin{array}{l}0 \\
\stackrel{0}{\circ}\end{array}$ & ' & , & 1 & $\left.\begin{array}{c}m \\
0\end{array}\right]$ & $v ?$ & $\tilde{0}$ & $\vec{i}$ & $\grave{0}$ & ' & & $\begin{array}{l}\overrightarrow{0} \\
\dot{0}\end{array}$ \\
\hline$\sum_{4}^{1}$ & $0^{\circ}$ & $\mid \begin{array}{l}0 \\
\infty \\
i \\
i n\end{array}$ & $\simeq$ & in & $\begin{array}{l}0 \\
\dot{\sim} \\
\sim\end{array}$ & in & in & 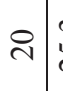 & $\begin{array}{l}n \\
n \\
n\end{array}$ & $\bar{m} \delta$ & $\therefore)$ & $\begin{array}{c}m \\
\stackrel{2}{r}\end{array}$ & \begin{tabular}{c|c}
6 & $m$ \\
$y$ \\
$y$
\end{tabular} & 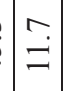 & $\mid \begin{array}{l}1 \\
\\
\end{array}$ & $\begin{array}{l}\infty \\
\dot{0} \\
\dot{b}\end{array}$ & $\stackrel{m}{\infty}$ & ชె & î. \\
\hline & 吾 & $\begin{array}{c}\infty \\
\infty \\
\infty\end{array}$ & $\underset{f}{J}$ & $\mid \begin{array}{l}\infty \\
\sim \\
\sim\end{array}$ & $\underset{\sim}{\stackrel{g}{e}}$ & $\stackrel{\infty}{\beth}$ & î & $\underset{\widetilde{N}}{\mathbb{N}}$ & 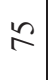 & $\cong$ & $\stackrel{0}{=} \frac{\alpha}{\tau}$ & $\frac{\infty}{m} \mid \frac{\vec{m}}{m}$ & $\begin{array}{l}n \\
n\end{array}$ & $\left|\begin{array}{l}\infty \\
0 \\
0\end{array}\right|$ & $\hat{i}$ & $\underset{\sim}{\mathbb{N}}$ & $\stackrel{n}{\sim}$ & 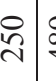 & $\stackrel{\infty}{+}$ \\
\hline & 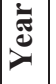 & $\tilde{\sigma}$ & よ̆ & $\stackrel{2}{\sigma}$ & $\widehat{\widehat{\sigma}}$ & $\widehat{\widehat{\sigma}}$ & $\stackrel{\infty}{2}$ & छे̀ & 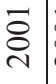 & 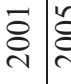 & ¿̊. & 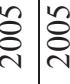 & 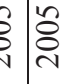 & $\left|\begin{array}{l}\stackrel{\Xi}{0} \\
\grave{ }\end{array}\right|$ & 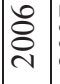 & 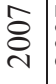 & હેे & 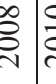 & \\
\hline & U & 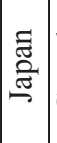 & 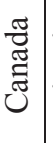 & 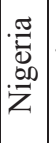 & 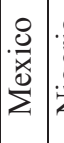 & 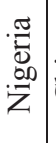 & : & 营 & . & 武 & 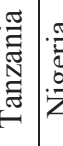 & 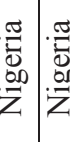 & 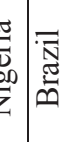 & 芯 & 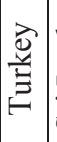 & 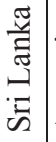 & 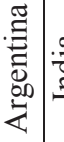 & $\Xi$ & \\
\hline & & 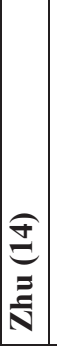 & 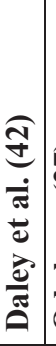 & 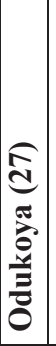 & 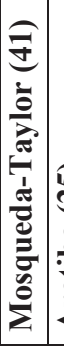 & 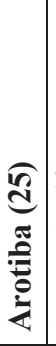 & 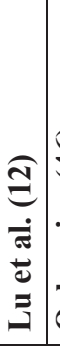 & 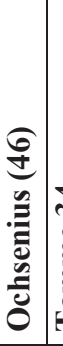 & 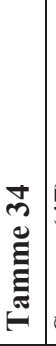 & 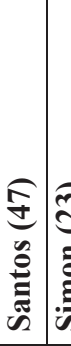 & 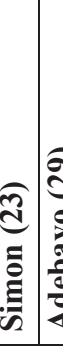 & 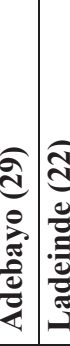 & 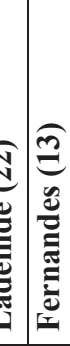 & 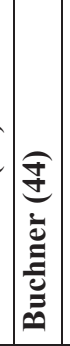 & 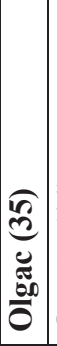 & 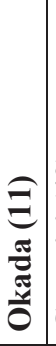 & 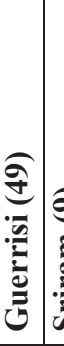 & 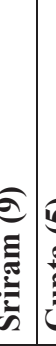 & 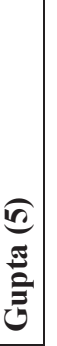 \\
\hline
\end{tabular}


Table 5. Gender and site distribution of odontogenic tumors with large studies reported from different countries and regions.

\begin{tabular}{|c|c|c|c|c|c|}
\hline Authors & Country & Continent & Cases (n) & M:F & Max: Mand \\
\hline $\begin{array}{l}\text { Mosqueda-Taylor } \\
\text { (39) }\end{array}$ & Mexico & America & 347 & $1: 1.25$ & $1.03 ; 1$ \\
\hline Buchner (35) & California & America & 1030 & $1.7: 1$ & $1: 1.13$ \\
\hline Ochsenius(44) & Chile & America & 362 & $1: 1.15$ & $1: 1.14$ \\
\hline Fernandes (42) & Brazil & America & 340 & $1: 1.23$ & $1: 1.90$ \\
\hline Santos (45) & Brazil & America & 127 & $1: 1.76$ & $1: 1.36$ \\
\hline Avela (41) & Brazil & America & 169 & $1: 1.35$ & $1: 1.77$ \\
\hline Olgac (33) & Turkey & Europe & 476 & $1: 1.06$ & $1: 1.94$ \\
\hline Tamme (32) & Estonia & Europe & 75 & $1: 1.68$ & $1: 1.68$ \\
\hline Adebayo (27) & $\mathrm{N}$ Nigeria & Africa & 318 & $1.35: 1$ & $1: 4.41$ \\
\hline Arotiba (23) & W Nigeria & Africa & 128 & $1.09: 1$ & $1: 2.88$ \\
\hline Ladeinde (20) & SW Nigeria & Africa & 319 & $1.03: 1$ & $1: 4.08$ \\
\hline Simon (21) & Tanzania & Africa & 111 & $1: 1.09$ & NS \\
\hline Sriram ${ }^{9}$ & W India & Asia & 250 & $1.19: 1$ & $1: 3.80$ \\
\hline Gupta $^{5}$ & S India & Asia & 489 & $1.08: 1$ & $1: 4.02$ \\
\hline Jing ${ }^{10}$ & W China & Asia & 1054 & $1.27: 1$ & $1: 3.83$ \\
\hline Luo $^{8}$ & N China & Asia & 802 & $1.14: 1$ & $1: 3.89$ \\
\hline Zhang (53) & Japan & Asia & 203 & $1.03: 1$ & $1: 2.27$ \\
\hline $\begin{array}{l}\text { Gaitán-Cepeda } \\
\text { (34) }\end{array}$ & Mexico & America & 136 & $1.14: 1$ & NS \\
\hline Gill (14) & Western India & Asia & 2,09 & $1.29: 1$ & $1: 2.26$ \\
\hline Tawfik (17) & Egypt & Africa & 82 & $1.2: 1$ & $0.2: 1$ \\
\hline Mamabolo (30) & South Africa & Africa & 743 & $1.06: 1$ & $1: 1.82$ \\
\hline da-Costa (48) & Brazil & America & 201 & $1.33: 1$ & $1: 2.69$ \\
\hline Servato (50) & Brazil & America & 240 & $1: 1.1$ & $1: 2.6$ \\
\hline Osterne (46) & Brazil & America & 185 & $1.24: 1$ & $1: 2.1$ \\
\hline El-Gehani (18) & Libya & Africa & 148 & $1.31: 1$ & $1: 2.08$ \\
\hline Ebenezer (14) & Chennai & Asia & 107 & $1: 1.6$ & $1: 2.45$ \\
\hline Siriwardena (16) & Sri Lanka & Asia & 1677 & $1.04: 1$ & $1: 2.8$ \\
\hline Wu and Chan (13) & Hong Kong & Asia & 68 & $1: 1.06$ & $1: 2.40$ \\
\hline Ogunsalu (43) & Jamaica & America & 80 & NS & $1: 7.75$ \\
\hline Saghravanian $^{7}$ & Iran & Asia & 165 & $1: 1.17$ & $1: 2.43$ \\
\hline Okada $^{11}$ & Sri Lanka & Asia & 226 & 1:1.11 & $1: 3.91$ \\
\hline Present Study & Turkey (Cappadocia) & Asia & 218 & $1.01: 1$ & $1: 3.54$ \\
\hline
\end{tabular}


the present study, this ratio was found as 5:1.Reichart et al (49) also reported that ameloblastomas are seen more frequently in the anterior region among Blacks (21.6\%) compared to Caucasians (12.6\%) and Asians (11.9\%). In the present series, the second most common odontogenic tumor was KCOT (26.15\%) and, in accordance with other series (50-52), it was responsible for nearly a quarter of the evaluated OT. This incidence was somewhat higher $(14,17,18,30)$, and somewhat lower $(8,10,15,34,41,46,48)$ than that seen in other series. This study also demonstrated that KCOT is rare in early childhood and has a strikingly higher prevalence during adolescence, when it was the most common OT.

Odontomas are an abnormal mass of calcified dental tissue, usually representing a developmental abnormality. Female patients were more affected than male patients in the present study, which is in agreement with reports from China (10), whereas Ladeinde et al. (20) in Nigeria reported no sex predilection in their study. In the present study, most odontomas were found in the posterior regions of both jaws. This finding was in accordance with many other reports from Mexico, Chile, Brazil, and Estonia (33,39,42,44).

Reports from African and Chinese populations generally present the highest frequency of malignant OT $(8,10,12,20,25)$, while studies from North and South America have informed rates of $1.6 \%$ and lower $(34,36,39,40,42,44)$ except for tertiary reference centers in the United States (37), Mexico (38) and the present study. In the present study, this ratio was found in 13 cases (4.59\%). Published reports also stated that malignant odontogenic tumors are rare and represent $0.1-6.1 \%$ of all tumors (Tables 3,4). The mean age of 64 years for malignant tumors in the present study is higher than in other studies in southern Asia (mean 46 y) and eastern Asia (mean 41 y) $(8-11,53)$. PIOC was the most malignant entity encountered in this analysis and represented $4.58 \%$ (10 cases) of odontogenic tumors. It was found to occur more in female patients and in the maxilla. The female predilection is in contrast to other reports $(19,20)$.

Site distribution of odontogenic tumors with large studies reported from different countries and regions is shown in table 5. The majority of studies confirm the mandible as the anatomic site most frequently affected by OTs, especially by ameloblastoma and KCOT, which agrees with our findings $(5,17,39,46)$. The preference for the mandible in this study, 1:3.52, is a mean between several studies in Nigeria and African countries $(19,20,25)$, which provide values of 2.9 to $5.7: 1$, in contrast to Americans $(39,41,44)$, and Europeans $(11,32)$, where lower values are observed in the jaw with ratios of 1 to $2: 1$. These values can be explained by the prevalence of AME being far greater in African countries. In present study, almost $83 \%$ of ameloblastomas were located in the mandible, with a very high mandible to maxilla ratio of 5:1. This is similar with the studies by Reichart et al. (49) who found, in an extensive review of all the cases reported in the literature, the ratio to be around 5.4:1. In the present study, ameloblastomas were frequently encountered in the molar-ramus region in the mandible and the molar region in the maxilla.

In relation to sociodemographic data, a higher proportion of males were affected with OT and the average age at diagnosis was 35 years (48). The gender distribution of odontogenic tumors in large studies reported from different countries and regions was shown in table 5. Avelar et al. (41) reported that male patients were more affected than female patients, agreeing with several studies from China $(10,12)$, Nigeria $(19,20)$, Egypt (17), India (9), and Canada (35). However, the preponderance of females was reported in Sri Lanka $(11)$, Brazil $(42,45)$, Mexico (39), Chile (44), Nigeria (25), and Estonia (32). In present study, an almost equal gender distribution was observed, with a slight predominance of males. The literature states that patients with OT are usually diagnosed in the second to fifth decades of life $(8,45)$, but the frequency of different lesions varies with the age of the patient. In this study, odontogenic tumors showed a peak incidence in the fourth decade of life, which was probably related to the high prevalence of $\mathrm{AME}$ and KCOT in this age group; there was a prevalence of odontomas in the second decade, while other studies described a high frequency of ameloblastomas and KCOT. In older patients, there is a predominance of ameloblastomas and KCOT $(8,10)$. Some studies reported that various types of OT, including AOT, odontoma, and calcifying cystic OT (CCOT), were more frequent in the second decade of life $(8,12,17)$.

In conclusion, the present study reflects not only differences in the distribution of odontogenic tumors but also similarities among the various population samples assessed both in Asia and around the world. These data are important to assess geographic differences in the incidence of lesions and to allow clinicians to make realistic judgments in counseling patients before biopsy about the probability of diagnosis and risks associated with nonspecific clinical or radiographic lesions. The incidences of OTs observed in the present study are similar to those in previous studies from Asia and Africa and in contrast to those reported from American and European countries.

\section{References}

1. Philipsen HP, Reichart PA. Revision of the 1992-edition of the WHO histological typing of odontogenic tumours. A suggestion. J Oral Pathol Med. 2002;31:253-8.

2. Mosqueda-Taylor A. New findings and controversies in odontogenic tumors. Med Oral Patol Oral Cir Bucal. 2008;13:E555-8.

3. Regezi JA, Kerr DA, Courtney RM. Odontogenic tumors: analysis of 706 cases. J Oral Surg. 1978;36:771-8.

4. Lawal AO, Adisa AO, Olusanya AA. Odontogenic tumors: a review of 266 cases. J Clin Exp Dent 2013;5:e13-7. 
5. Gupta B, Ponniah I. The pattern of odonto-genic tumors in a government teaching hos-pital in the southern Indian state of Tamil Nadu. Oral Surg Oral Med Oral Pathol Oral Radiol Endod. 2010;110:32-9. 6. MacDonald-Jankowski DS, Li TK. Keratocystic odontogenic tumour in a Hong Kong community: the clinical and radiological features. Dentomaxillofac Radiol. 2010;39:167-75.

7. Saghravanian N, Jafarzadeh $H$, Bashardoost N, Pahlavan N, Shirinbak I. Odontogenic tumors in an Iranian population: a 30-year evaluation. J Oral Sci. 2010;52:391-6.

8. Luo HY, Li TJ. Odontogenic tumors: a study of 1309 cases in a Chinese population. Oral Oncol. 2009;45:706-11.

9. Sriram G, Shetty RP. Odontogenic tumors: a study of 250 cases in an Indian teaching hospital. Oral Surg Oral Med Oral Pathol Oral Radiol Endod. 2008;105:14-21.

10. Jing W, Xuan M, Lin Y, Wu L, Liu L, Zheng X, et al. Odontogenic tumours: a retrospective study of 1642 cases in a Chinese population. Int J Oral Maxillofac Surg. 2007;36:20-5.

11. Okada H, Yamamoto H, Tilakaratne WM. Odontogenic tumors in Sri Lanka: analysis of 226 cases. J Oral Maxillofac Surg. 2007;65:875-82.

12. Lu Y, Xuan M, Takata T, Wang C, He Z, Zhou Z, et al. Odontogenic tumors. A demographic study of 759 cases in a Chinese population. Oral Surg Oral Med Oral Pathol Oral Radiol Endod. 1998;86:707-14.

13. Wu PC, Chan KW. A survey of tumours of the jawbones in Hong Kong Chinese: 1963-1982. Br J Oral Maxillofac Surg. 1985;23:92102.

14. Ebenezer V, Ramalingam B. A cross-sectional survey of prevalence of odontogenic tumours. J Maxillofac Oral Surg. 2010;9:36974.

15. Varkhede A, Tupkari JV, Sardar M. Odontogenic tumors: a study of 120 cases in an Indian teaching hospital. Med Oral Patol Oral Cir Bucal. 2011;16:e895-9.

16. Siriwardena BS, Tennakoon TM, Tilakaratne WM. Relative frequency of odontogenic tumors in Sri Lanka: Analysis of 1677 cases. Pathol Res Pract. 2012;208:225-30.

17. Tawfik MA, Zyada MM. Odontogenic tumors in Dakahlia, Egypt: analysis of 82 cases. Oral Surg Oral Med Oral Pathol Oral Radiol Endod. 2010;109:67-73.

18. El-Gehani R, Orafi M, Elarbi M, Subhashraj K. Benign tumours of orofacial region at Benghazi, Libya: a study of 405 cases. J Craniomaxillofac Surg. 2009;37:370-5.

19. Adebayo ET, Ajike SO, Adekeye EO. A review of 318 odontogenic tumors in Kaduna, Nigeria. J Oral Maxillofac Surg. 2005;63:811-9.

20. Ladeinde AL, Ajayi OF, Ogunlewe MO, Adeyemo WL, Arotiba GT, Bamgbose BO, et al. Odontogenic tumors: a review of 319 cases in a Nigerian teaching hospital. Oral Surg Oral Med Oral Pathol Oral Radiol Endod. 2005;99:191-5.

21. Simon EN, Merkx MA, Vuhahula E, Ngassapa D, Stoelinga PJ. A 4-year prospective study on epidemiology and clinicopathological presentation of odontogenic tumors in Tanzania. Oral Surg Oral Med Oral Pathol Oral Radiol Endod. 2005;99:598-602.

22. Simon EN, Stoelinga PJ, Vuhahula E, Ngassapa D. Odontogenic tumours and tumour- like lesions in Tanzania. East Afr Med J. 2002;79:3-7.

23. Arotiba JT, Ogunbiyi JO, Obiechina AE. Odontogenic tumours: a 15-year review from Ibadan, Nigeria. Br J Oral Maxillofac Surg. 1997;35:363-7.

24. Chidzonga MM, Lopez VM, Alverez AP. Odontogenic tumours: analysis of 148 cases in Zimbabwe. Cent Afr J Med. 1996;42:158-61. 25. Odukoya O. Odontogenic tumors: analysis of 289 Nigerian cases. J Oral Pathol Med. 1995;24:454-7.

26. Mosadomi A. Odontogenic tumors in an African population. Analysis of twenty-nine cases seen over a 5-year period. Oral Surg Oral Med Oral Pathol. 1975;40:502-21.

27. Adebayo ET, Ajike SO, Adekeye EO. Odontogenic tumours in children and adolescents: a study of 78 Nigerian cases. J Craniomaxillofac Surg. 2002;30:267-72.
28. Ajayi OF, Ladeinde AL, Adeyemo WL, Ogunlewe MO. Odontogenic tumors in Nigerian children and adolescents-a retrospective study of 92 cases. World J Surg Oncol. 2004;2:39.

29. Philipsen HP, Reichart PA, Praetorius F. Mixed odontogenic tumours and odontomas. Considerations on interrelationship. Review of the literature and presentation of 134 new cases of odontomas. Oral Oncol. 1997;33:86-99.

30. Mamabolo M, Noffke C, Raubenheimer E. Odontogenic tumours manifesting in the first two decades of life in a rural African population sample: a 26 year retrospective analysis. Dentomaxillofac Radiol. 2011;40:331-7.

31. Pippi R. Benign odontogenic tumours: clinical, epidemiological and therapeutic aspects of a sixteen years sample. Minerva Stomatol. 2006;55:503-13.

32. Tamme T, Soots M, Kulla A, Karu K, Hanstein SM, Sokk A, et al. Odontogenic tumours, a collaborative retrospective study of 75 cases covering more than 25 years from Estonia. J Craniomaxillofac Surg. 2004;32:161-5.

33. Olgac V, Koseoglu BG, Aksakalli N. Odontogenic tumors in Istanbul: 527 cases. Br J Oral Maxillofac Surg. 2006;44:386-388.

34. Gaitan-Cepeda LA, Quezada-Rivera D, Tenorio-Tocha F, Leyva-Huerta ER. Reclas-sification of odontogenic keratocyst as tumour. Impact on the odontogenic tumours prevalence. Oral Dis. 2010;16:185-7.

35. Buchner A, Merrell PW, Carpenter WM. Relative frequency of central odontogenic tumors: a study of 1,088 cases from Northern California and comparison to studies from other parts of the world. J Oral Maxillofac Surg. 2006;64:1343-52.

36. Buchner A, Merrell PW, Carpenter WM. Relative frequency of peripheral odonto-genic tumors: a study of 45 new cases and comparison with studies from the literature. J Oral Pathol Med. 2006;35:385-91.

37. Goldenberg D, Sciubba J, Koch W, Tufano RP. Malignant odontogenic tumors: a 22-year experience. Laryngoscope. 2004:114:1770-4.

38. Mosqueda-Taylor A, Meneses-Garcia A, Ruiz-Godoy Rivera LM, Suarez Roa ML, Luna Ortiz K. Malignant odontogenic tumors. A retrospective and collaborative study of seven cases. Med Oral. 2003;8:110-21.

39. Mosqueda-Taylor A, Ledesma-Montes C, Caballero-Sandoval S, Portilla-Robertson J, Ruíz-Godoy Rivera LM, Meneses-García A. Odontogenic tumors in Mexico: a collaborative retrospective study of 349 cases. Oral Surg Oral Med Oral Pathol Oral Radiol Endod. 1997:84:672-5.

40. Daley TD, Wysocki GP, Pringle GA. Relative incidence of odontogenic tumors and oral and jaw cysts in a Canadian population. Oral Surg Oral Med Oral Pathol. 1994;77:276-80.

41. Avelar RL, Antunes AA, Santos TS, Andrade ES, Dourado E. Odontogenic tumors: clinical and pathology study of 238 cases. Braz J Otorhinolaryngol. 2008;74:668-73.

42. Fernandes AM, Duarte EC, Pimenta FJ, Souza LN, Santos VR, Mesquita RA, et al. Odontogenic tumors: a study of 340 cases in a Brazilian population. J Oral Pathol Med. 2005;34:583-7.

43. Ogunsalu CO. Odontogenic tumours from two centres in Jamaica. A 15-year review. West Indian Med J. 2003;52:285-9.

44. Ochsenius G, Ortega A, Godoy L, Penafiel C, Escobar E. Odontogenic tumors in Chile: a study of 362 cases. J Oral Pathol Med. 2002;31:415-20.

45. Santos JN, Pinto LP, de Figueredo CR, de Souza LB. Odontogenic tumors: analysis of 127 cases. Pesqui Odontol Bras. 2001;15:308-13. 46. Osterne RL, Brito RG, Alves AP, Cavalcante RB, Sousa FB. Odontogenic tumors: a 5-year retrospective study in a Brazilian population and analysis of 3406 cases reported in the literature, Oral Surg. Oral Med. Oral Pathol. Oral Radiol. Endod. 2011;111:474-481. 47. Guerrisi M, Piloni MJ, Keszler A. Odontogenic tumors in children and adolescents. A 15-year retrospective study in Argentina. Med Oral Patol Oral Cir Bucal. 2007;12:E180-5. 
48. da-Costa DO, Maurício AS, de-Faria PA, da-Silva LE, MosquedaTaylor A, Lourenço SD. Odontogenic tumors: a retrospective study of four Brazilian diagnostic pathology centers. Med Oral Patol Oral Cir Bucal. 2012;17:e389-94.

49. Reichart PA, Philipsen HP, Sonner S. Ameloblastoma: biological profile of 3677 cases. Eur J Cancer B Oral Oncol. 1995;31B:86-99.

50. Servato JP, de Souza PE, Horta MC, Ribeiro DC, de Aguiar MC, de Faria PR, et al. Odontogenic tumours in children and adolescents: a collaborative study of 431 cases. Int J Oral Maxillofac Surg. 2012;41:768-73

51. Lima Gda S, Fontes ST, de Arau' jo LM, Etges A, Tarquinio SB, Gomes AP. A survey of oral and maxillofacial biopsies in children: a single-center retrospective study of 20 years in Pelotas-Brazil. J Appl Oral Sci. 2008;16:397-402.

52. Elarbi M, El-Gehani R, Subhashraj K, Orafi M. Orofacial tumors in Libyan children and adolescents. A descriptive study of 213 cases. Int J Pediatr Otorhinolaryngol. 2009;73:237-42.

53. Zhang M, Fukuyama H, Matsuo K, Yamashita Y, Hirashima S, Takahashi T, et al. Clinico-pathological analysis of odontogenic tumors according to the revised WHO (2005) histopathological classification in Japanese. J Kyushu Dent Soc. 2007;61:55-66.

\section{Conflict of Interest}

All authors declare they have no conflict of interests. 